REVIEW ARTICLE

\title{
A Review of Radiographic Imaging Findings of Ependymal Tumors
}

\author{
Maria Habib Hanna, $M D^{1^{*}}$, Bansal $A, M D^{2}$ and Belani $P, M D^{2}$ \\ ${ }^{1}$ Department of Radiology, Weill Cornell Medicine, New York, USA \\ ${ }^{2}$ Department of Radiology, Virginia Tech Carilion School of Medicine, Carilion Roanoke Memorial \\ Hospital, 1906 Belleview Ave SE, Roanoke, VA 24014, USA
}

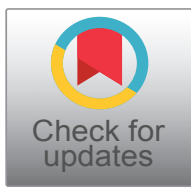

*Corresponding author: Maria Habib Hanna, MD, Assistant Professor, Department of Radiology, Weill Cornell Medicine, 525 E 68th St, NY 10065, New York, USA

\begin{abstract}
Ependymomas are glial tumors that typically arise from the lining of the ventricles or the central canal of the spinal cord. The most common site of occurrence is within the posterior fossa. Subtypes of ependymomas include anaplastic ependymoma, myxopapillary ependymoma, and subependymoma. Its characteristic imaging features include findings of a heterogeneous mass with necrosis, calcifications, cystic changes, and hemorrhage. Treatment options includes partial resection with or without irradiation.
\end{abstract}

\section{Introduction}

Ependymomas are rare glial tumors that account for approximately $7 \%$ of all intracranial neoplasms in adults [1]. Pediatric ependymomas are the $3^{\text {rd }}$ most common brain tumor in children. More than half of cases occur in children under the age of five [2].

They are most commonly found within the posterior fossa $(60 \%)$, followed by the supratentorial region (30\%) and spinal canal (10\%) [3]. The World Health Organization (WHO) classification system of ependymomas ranges from WHO Grade I-III depending on the location and histologic appearance [1]. Recent data supports the concept that supratentorial ependymomas have a different clinical and radiologic presentation than their infratentorial counterparts. The clinical and radiologic presentation is useful in narrowing the differential diagnosis of these brain tumors.

Although there have been further advancements in understanding the biomechanics and genomics of this entity, to date, there are no distinguished radiographic findings that can determine the specific subtype solely based on imaging. There are, however, imaging features that can typically be distinguished between the adult and pediatric patients. For instance, ependymomas in the adult population are typically larger than $4 \mathrm{~cm}$ in size when they present and often contain cystic components, whereas ependymomas in the pediatric population are usually solid and usually smaller in size at presentation [4].

Radiographic features of intracranial ependymomas are summarized in Table 1.

The types of ependymomas to be discussed in this article include intracranial ependymomas (infra and supratentorial) and spinal (myxopapillary ependymoma).

\section{Intracranial ependymoma}

Intracranial ependymomas are more commonly WHO Grade II and occur in both children and adults. Infratentorial ependymomas most often arise in children as posterior fossa tumors, [4] whereas supratentorial ependymomas are typically seen in younger to middle age adults [5].

\section{CT and MRI characteristics}

There are similar computed tomography (CT) and magnetic resonance imaging (MRI) imaging characteristics in both infratentorial and supratentorial ependymomas [4]. Although CT or MRI can be used to evaluate intracranial and spinal ependymomas, MRI is preferred because of its enhanced contrast resolution and the ability to better define the extent of tumor. However, CT can be useful in the evaluation of subtle calcifications which can present in up to $50 \%$

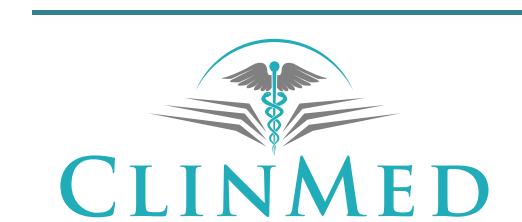

INTERNATIONAL LIBRARY

Citation: Hanna MH, Bansal A, Belani P (2019) A Review of Radiographic Imaging Findings of Ependymal Tumors. Neurosurg Cases Rev 2:028. doi.org/10.23937/2643-4474/1710028

Accepted: October 22, 2019; Published: October 24, 2019

Copyright: (c) 2019 Hanna MH, et al. This is an open-access article distributed under the terms of the Creative Commons Attribution License, which permits unrestricted use, distribution, and reproduction in any medium, provided the original author and source are credited. 
Table 1: Adult versus Pediatric intracranial ependymoma [16].

\begin{tabular}{|l|l|l|}
\hline \multicolumn{2}{|l|}{ Adults } & Pediatrics \\
\hline Age & Middle age adults & Children less than 5 years \\
\hline Size & $>4 \mathrm{~cm}$ & $<4 \mathrm{~cm}$ \\
\hline Location & Supratentorial & Infratentorial \\
\hline Imaging features & Cystic component & Solid component, Necrosis, Valcification \\
\hline
\end{tabular}

Typical findings in both the adult and pediatric population.

\begin{tabular}{|l|l|}
\hline CT features & Iso to slightly hypoattenuating, +Enhancement on post-contrast images \\
\hline MRI features & $\begin{array}{l}\text { T1 Isointense, T2 Hypointense } \\
\text { +Enhancement on Post-contrast images } \\
\text { Hypointense T1/T2 Foci-hemosiderin, Calcifications, or Necrosis }\end{array}$ \\
\hline
\end{tabular}

Based on a retrospective study of CT and MRI characteristics of 41 cases [17].

Table 2: CT Imaging findings of Intracranial ependymoma [17].

Solid components, large peripheral cystic component, 3-10 cm in size, Intraparenchymal supratentorial ependymoma was found mostly in left frontal and parietal lobes (18\% and $37 \%$ respectively).

Solid component-lobulated, well defined margins, mostly ISODENSE to brain parenchyma.

Table 3: MR Imaging findings of Intracranial ependymoma [16].

Heterogeneous in signal intensity, majority isointense on T1 and hypointense on T2, moderate to intense enhancement on post-contrast imaging.

Areas of diffusion restriction likely correspond to increased cellularity.

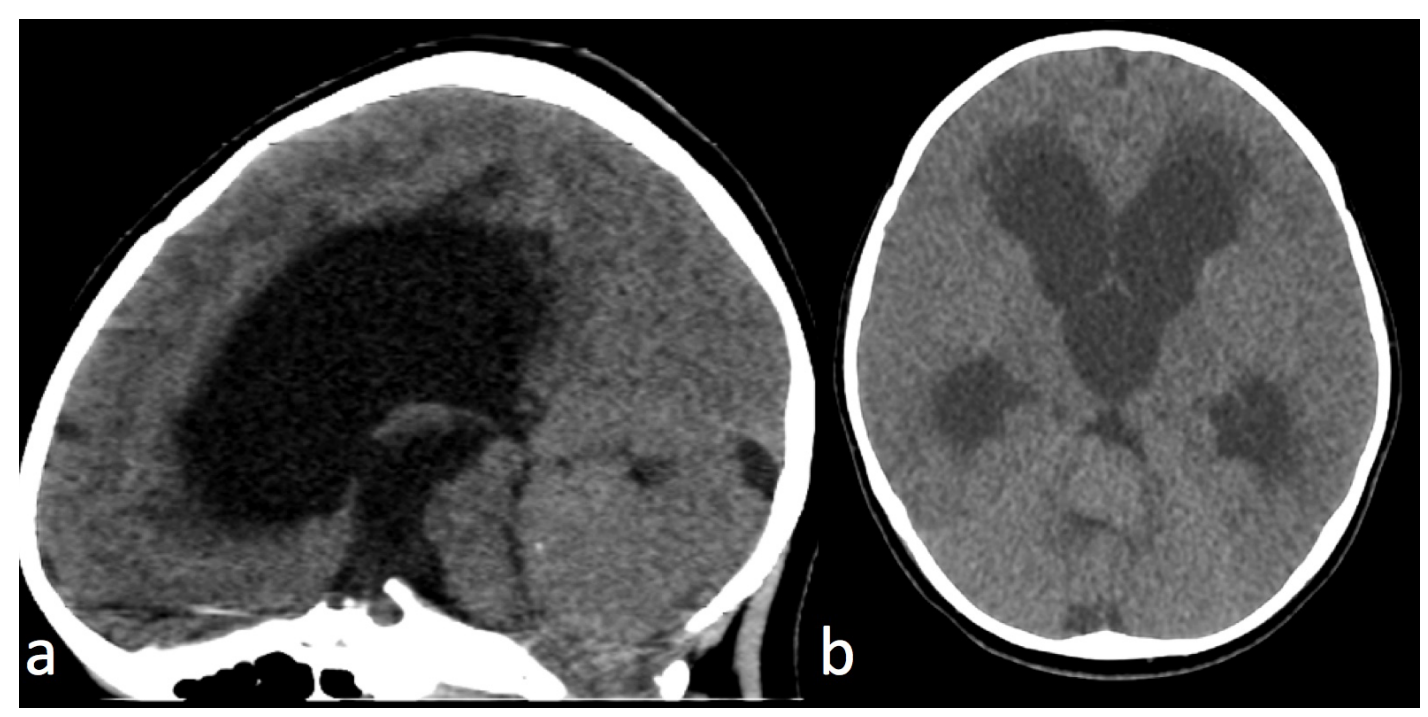

Figure 1: CT of intracranial infratentorial Ependymoma.

Non contrast CT Head a) Sagittal view; b) Axial view.

3-year-old with truncal ataxia. Non contrast CT head demonstrates a large isodense posterior fossa mass originating from the fourth ventricle and resulting in severe hydrocephalus with transependymal CSF absorption. A punctate calcification is present in the superior portion of the mass.

of lesions [1]. CT findings of ependymoma include hyperdense soft tissue components, calcifications, and hemorrhage [6]. The tumor typically enhances on post-contrast imaging (Table 2).

The MRI features of ependymomas include low T1, high T2, and iso- to high fluid-attenuated inversion recovery (FLAIR) signal intensity due to their increased myxoid component [7]. Ependymomas are heterogeneously enhancing masses that demonstrate avid enhancement of the soft tissue components with ad- jacent areas of little to no enhancement. In the posterior fossa, ependymomas typically arise in the floor of the fourth ventricle and the pliable nature of these tumors can result in extension of the lesion from the fourth ventricle through the foramen of Luschka or foramen of Magendie [6] (Figure 1 and Figure 2). See Table 3.

\section{Anaplastic ependymoma}

Anaplastic ependymoma is a WHO Grade III intra- 


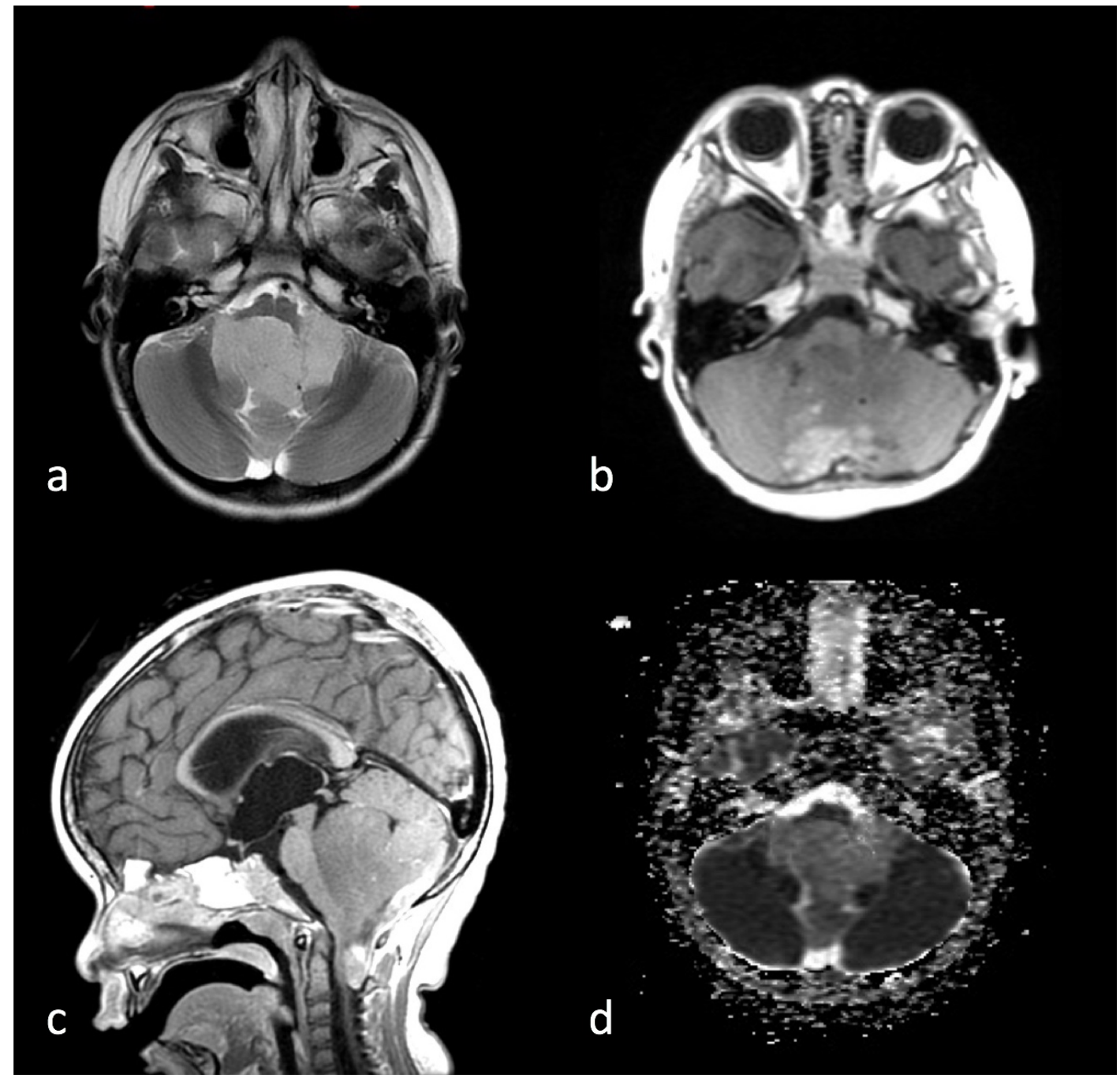

Figure 2: MRI of intracranial infratentorial Ependymoma (same patient as in Figure 1).

MRI Brain a) Axial T1 post-contrast; b) Axial T2 FSE; c) Sagittal T1 with contrast; d) Axial ADC map.

A large mass predominantly filling and expanding the fourth ventricle with extension of the lesion through the foramen of Luschka and Magendie. The lesion was low to isointense on T1 and hyperintense on T2 weighted images. There was no restricted diffusion.

cranial tumor (typically supratentorial) that is more aggressive with a higher proliferative rate and a greater tendency for infiltration and dissemination into the cerebrospinal fluid [8]. It is a rare tumor and its criteria for histopathologic grading is not well established, however includes marked hypercellularity, nuclear atypia, and increased mitotic activity.

Imaging characteristics include a heterogeneous intraparenchymal mass with both cystic and solid components. There is heterogeneous enhancement on both $\mathrm{CT}$ and MRI [9]. Calcifications can be seen on CT within both the solid and cystic regions of the mass.

MRI features include a heterogeneous appearance on both T1/T2 weighted imaging related to foci of hemorrhage, necrosis, calcification, and increased vascularity [10]. The tumor may demonstrate high signal on diffusion weighted images (DWI) with associated low signal on apparent diffusion coefficient (ADC) maps due to the increased cellularity of the tumor (Figure 3 ).
The clinical manifestations of anaplastic ependymoma are similar to findings that can be seen with WHO Grade II ependymoma and relate to increased intracranial pressure. Anaplastic ependymomas may be infiltrative within the brain or spinal cord and usually portend a poorer prognosis [8].

\section{Spinal ependymoma}

Although rare, ependymomas may arise in the spine. They typically present in adults with a mean age of 39 years and are more common in males [2]. The only known genetic disorder to be associated with intramedullary ependymoma is neurofibromatosis type 2 [6].

Spinal ependymomas have a central location within the cord, well defined margins, and can be associated with syringohydromyelia [11]. See Table 4. Spinal ependymomas are usually located within the central cord and have poorly delineated margins. If not completely excised, it results in a poorer prognosis. CSF dissemination is common in this entity, which further 


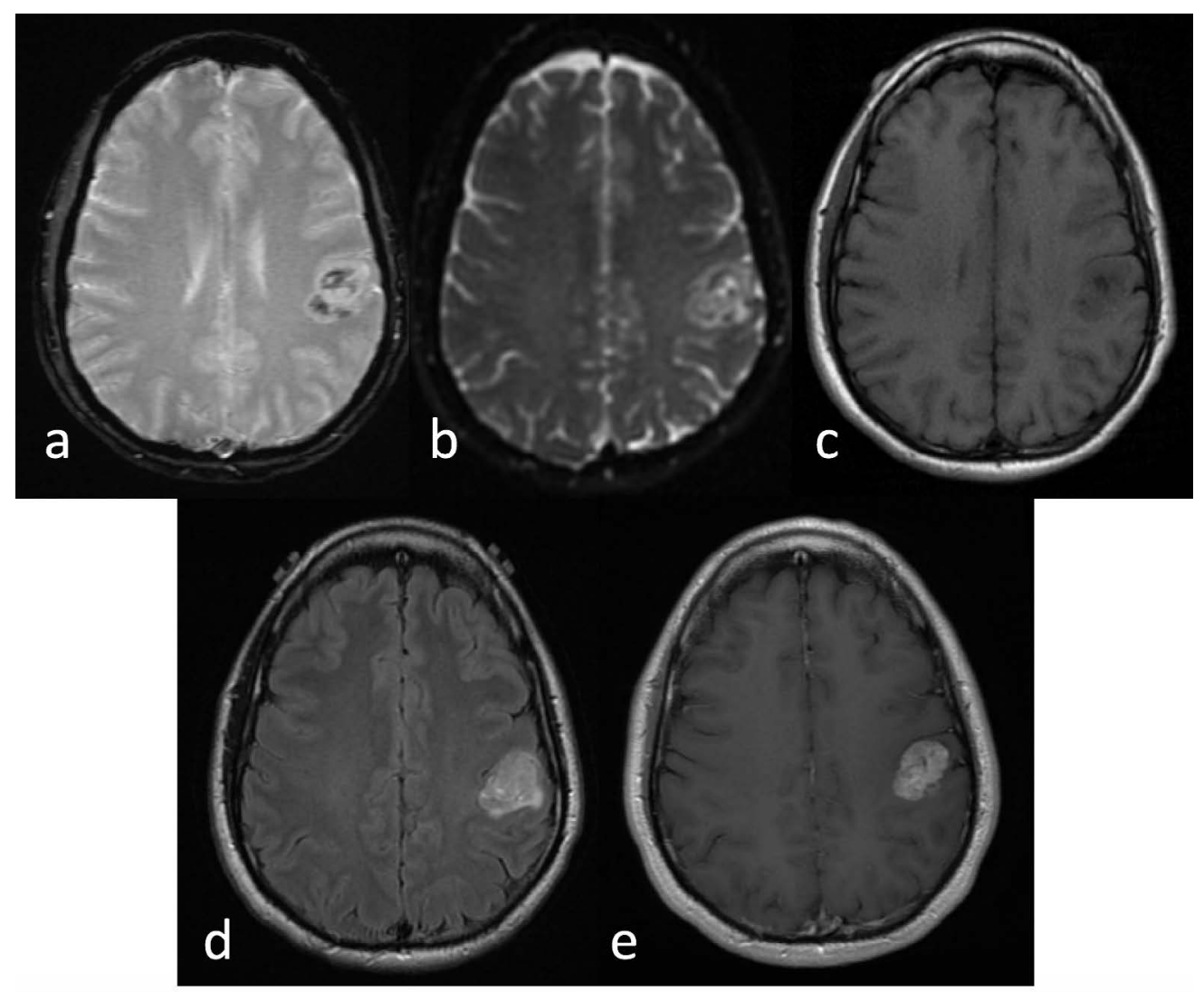

Figure 3: Anaplastic ependymoma.

MRI Brain a) Axial gradient recalled echo; b) Axial ADC map; c) Axial T1 pre contrast; d) Axial T2-Flair; e) Axial T1 post-contrast.

35-year-old left handed man presented with 6 months of progressive right thumb and facial numbness. MRI brain reveals a lobular and avidly enhancing left parietal mass centered in the left post central gyrus, with slight mass effect on the pre central gyrus. There were foci of susceptibility and diffusion restriction within the mass.

Table 4: Spinal ependymoma [18].

Subtypes: Cellular, Clear cell, Papillary, Tancytic ependymoma

\begin{tabular}{l|l} 
Neuroimaging findings & Well circumscribed, variable enhancement, may have cystic components, hemorrhage,
\end{tabular} calcification.

Prognosis Poor prognosis due to CSF dissemination.

portends a poor prognostic factor. The differential diagnosis for spinal ependymoma is astrocytoma. Ependymomas are more likely to be located centrally within the cord and have sharper margins of the tumor when compared to astrocytomas [7].

They are iso- to hypointense on T1 weighted images and hyperintense on T2 weighted imaging. A cap sign, referring to low $\mathrm{T} 2$ signal intensity along the margins of the tumor, may be seen with spinal ependymomas and relates to hemosiderin deposition above or below the tumor from chronic hemorrhage of the lesion [9]. There can be considerable edema surrounding the tumor, best seen on the T2 weighted images. On diffusion weighted images, there is reduced diffusion, which relates to the tissue composition of ependymomas and their high cellularity [6] (Figure 4).

Ependymomas within the spine cause expansion of the spinal canal due to their intramedullary location [2]. There may be associated vertebral body scalloping, pedicle erosion, or laminar thinning [11].

\section{Advanced imaging}

When advanced imaging is available, such as perfusion MRI, imaging findings may demonstrate elevated cerebral blood volume within the tumor. Proton MR spectroscopy may reveal elevated choline and reduced $\mathrm{N}$-acetylaspartate [7]. Diffusion tensor imaging (DTI) or tractography is a noninvasive method to estimate the direction and integrity of white matter tracts and has been used in evaluating spinal ependymoma. DTI has been used as an adjunct to conventional imaging to determine the location of tumor margins and degree of deflection of white matter tracts [12]. Astrocytomas are typically infiltrative and tend to disrupt the white matter tracts, whereas ependymomas tend to displace the white matter tracts [12]. 

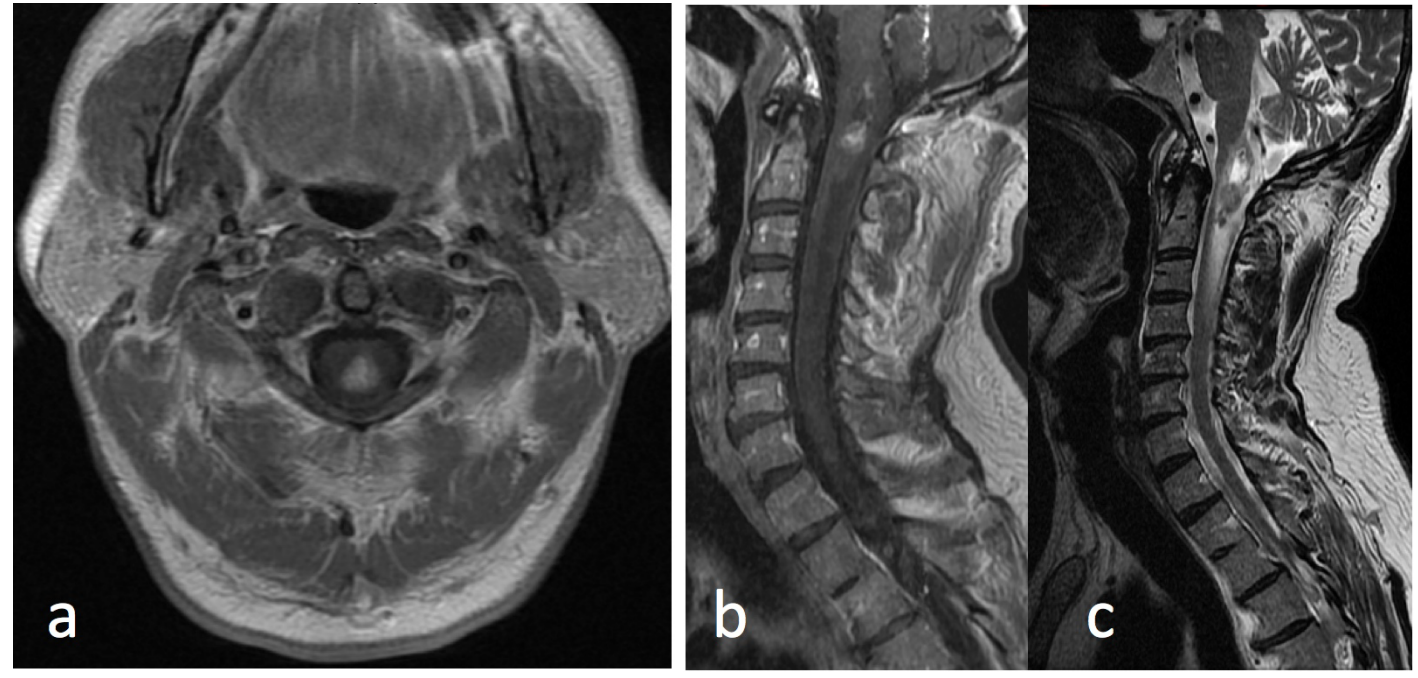

Figure 4: Spinal ependymoma.

MRI Cervical Spine: a) Axial T1 post-contrast at the level of the upper cervical spine; b) Sagittal T1 post-contrast; c) Sagittal T2 FSE.

61-year-old male with history of bilateral vestibular schwanomas and a heterogeneously enhancing mass within the upper cervical cord centrally with surrounding edema. Constellation of findings is consistent with Neurofibromatosis type II and the spinal lesion is a presumed spinal ependymoma.
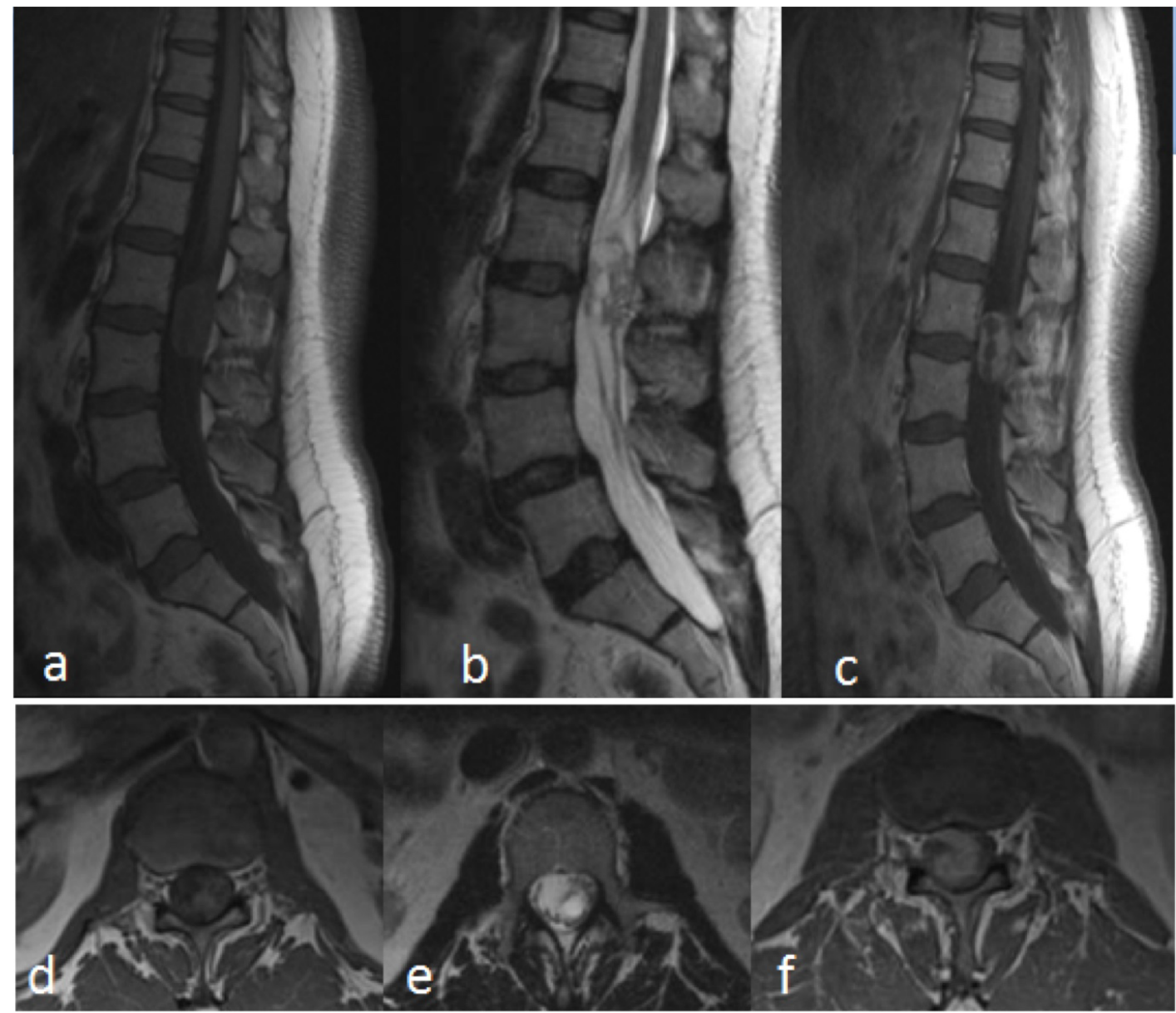

Figure 5: Myxopapillary ependymoma.

MRI Lumbar spine a) Sagittal T1; b) Sagittal T2 FSE; c) Sagittal T1 post-contrast; d) Axial T1; e) Axial T2 FSE; f) Axial T1 post-contrast.

59-year-old woman, presents with increasing pain radiating down the right leg, some bladder hesitancy, but no weakness of lower extremities. MRI of the lumbar spine shows a $5 \times 2 \times 1.5 \mathrm{~cm}$ heterogeneously enhancing mass with cystic components. The tumor was located in the filum terminale from L1 to L3. Surgical pathology was consistent with myxopapillary ependymoma. 


\section{Myxopapillary ependymoma}

Myxopapillary Ependymomas are WHO Grade I tumors and a more indolent variant of ependymoma that arise from the ependymal glial cells of the filum terminale [13] and predominately present in young males. They are the most common neoplasm found within the filum terminale [13]. Given their location within the spinal canal, lower back pain and/or weakness are common symptoms.

Myxopapillary ependymomas are typically lobular, often encapsulated, and contain gelatinous material [13]. They may have cyst formation and hemorrhage [13]. These typically occur in the extradural space, [2] compared to the intramedullary location of other spinal ependymomas. These lesions are best characterized on MRI imaging and are usually isointense relative to the spinal cord on T1 weighted images and hyperintense on T2 weighted images [14]. If there is hyperintensity within the lesion on both $\mathrm{T} 1$ and $\mathrm{T} 2$ weighted images, it likely reflects its mucin or hemorrhagic content. Myxopapillary ependymomas enhance after the administration of intravenous gadolinium. The location of these tumors within the conus medullaris suggests this diagnosis [15] (Figure 5).

\section{Discussion}

Ependymomas are a group of glial tumors that arise from the lining of the ventricles of the brain or the central canal. Ependymomas can occur at any age. $40 \%$ of ependymomas are supratentorial while $60 \%$ are infratentorial in location.

Radiologic imaging plays a role in both the diagnostic workup and treatment of patients with ependymoma. MRI is the preferred imaging modality due to lack of ionizing radiation, particularly when imaging the pediatric population. Additionally, MRI allows for better delineation of the tumor. Some lesions demonstrates areas of necrosis and calcifications.

Although there has been an improved understanding of varying subtypes of the ependymomas based on molecular biochemistry, there are no distinct imaging characteristics to date that has shown to be able to define such subtypes.

\section{Conclusion}

In conclusion, although rare, ependymomas can present in a variety of appearances and locations, some of which are classic such as a pliable $4^{\text {th }}$ ventricular tumor in a pediatric patient or an enhancing mass of the filum terminale in an adult. Knowing the classic appearance of the various types of ependymomas will help guide clinical management.

\section{References}

1. McLendon RE, Wiestler OD, Kros JM, Korshunov A, Ng HK (2016) Ependymoma. WHO classification of tumours of the central nervous system. (4 ${ }^{\text {th }}$ edn), International Agency for Research on Cancer, Lyon.

2. Koeller KK, Sandberg GD (2002) Cerebral intraventricular neoplasms: Radiologic-pathologic correlation. Radiographics 22: 1473-1505.

3. Howlader N, Noone AM, Krapcho M, Miller D, Bishop K, et al. (2016) SEER cancer statistics review, 1975-2014. National Cancer Institute.

4. Mermuys K, Jeuris W, Vanhoenacker PK, Van Hoe L, D'Haenens P (2005) Supratentorial ependymoma. AFIP Archives - Best Cases from the AFIP.

5. Smith AB, Smirniotopoulos JG, Horkanyne-Szakaly I (2013) From the radiologic pathology archives: Intraventricular neoplasms: Radiologic-pathologic correlation. Radiographics 33: 21-43.

6. Plaza MJ, Borja MJ, Altman N, Saigal G (2013) Conventional and advanced mri features of pediatric intracranial tumors: Posterior fossa and suprasellar tumors. AJR Am J Roentgenol 200: 1115-1124.

7. Vitanza NA, Partap S (2015) Pediatric ependymoma. Journal of Child Neurology 31: 1354-1366.

8. McLendon RE, Wiestler OD, Kros JM, Korshunov A, Ng HK (2016) Subependymoma. WHO classification of tumours of the central nervous system. ( $4^{\text {th }}$ edn), International Agency for Research on Cancer, Lyon.

9. Kim DH, Kim JH, Choi SH, Sohn CH, Yun TJ, et al. (2014) Differentiation between intramedullary spinal ependymoma and astrocytoma: Comparative MRI analysis. Clin Radiol 69: 29-35.

10. Tominaga $T$, Kayama $T$, Kumabe $T$, Sonoda $Y$, Yoshimoto T (1995) Anaplastic ependymomas: Clinical features and tumour suppressor gene p53 analysis. Acta Neurochir (Wien) 135: 163-170.

11. Ferrante L, Mastronardi L, Celli $P$, Lunardi $P$, Acqui M, et al. (1992) Intramedullary spinal cord Ependymomas: A study of 45 cases with long-term follow-up. Acta Neurochir 119: 74-79.

12. Choudhri AF, Whitehead MT, Klimo P, Montgomery BK, Boop FA (2014) Diffusion tensor imaging to guide surgical planning in intramedullary spinal cord tumors in children. Neuroradiology 56: 169-174.

13. McLendon RE, Rosenblum MK, Schiffer D, Wiestler OD (2016) Myxopapillary ependymoma. WHO classification of tumours of the central nervous system. ( $4^{\text {th }}$ edn $)$, International Agency for Research on Cancer, Lyon.

14. Lehman N (2008) Patterns of brain infiltration and secondary structure formation in supratentorial ependymal tumors. J Neuropathol Exp Neurol 67: 900-910.

15. Koeller KK, Rosenblum RS, Morrison AL (2000) Neoplasms of the spinal cord and filumterminale: Radiologic-pathologic correlation. Radiographics 20: 1721-1749.

16. Yuh EL, Barkovich AJ, Gupta N (2009) Imaging of ependymomas: MRI and CT. Childs Nerv Syst 25: 1203-1213.

17. Mangalore S, Aryan S, Prasad C, Santosh V (2015) Imaging characteristics of supratentorial ependymomas: Study on a large single institutional cohort with histopathological correlation. Asian J Neurosurg 10: 276-281.

18. Abdelzaher E (2018) Ependymoma. Pathology Outlines. com website. 